\title{
Circular RNA SLC26A4 regulates the maturation of microRNA-15a in non-small cell lung cancer cells
}

\author{
QIANKUN CHEN ${ }^{1}, \mathrm{HUA} \mathrm{LI}^{2}$ and $\mathrm{JI} \mathrm{LIU}^{2}$ \\ ${ }^{1}$ Department of Thoracic Surgery, Shanghai Pulmonary Hospital, Tongji University; \\ ${ }^{2}$ Department of Anesthesiology, Shanghai Pulmonary Hospital, Shanghai 200433, P.R. China
}

Received December 8, 2020; Accepted April 15, 2021

DOI: 10.3892/ol.2021.12983

\begin{abstract}
To the best of our knowledge, the oncogenic role of circular RNA solute carrier family 26 member 4 (circSLC26A4) has only been reported in cervical cancer, while its role in non-small cell lung cancer (NSCLC) is unknown. The present study explored the involvement of circSLC26A4 in NSCLC. NSCLC tissues and paired adjacent non-tumor tissues were collected from 64 patients with NSCLC. The expression levels of circSLC26A4, mature microRNA-15a (miR-15a) and miR-15a precursor in these tissues were determined by reverse transcription-quantitative PCR (RT-qPCR). NSCLC cells were transfected with pcDNA3.1-circSLC26A4 vector to overexpress circSLC26A4, followed by the measurement of the expression levels of mature miR-15a and miR-15a precursor using RT-qPCR. Cell proliferation was analyzed using a Cell Counting Kit-8 assay. circSLC26A4 expression was upregulated in NSCLC tissues, and its high expression was significantly associated with poor survival of patients with NSCLC. The expression levels of circSLC26A4 were correlated with the expression levels of mature miR-15a, but not the expression levels of miR-15a precursor in NSCLC tissues. In NSCLC cells, overexpression of circSLC26A4 was associated with downregulation of mature miR-15a expression, but not miR-15a precursor expression. A cell proliferation assay revealed that overexpression of circSLC26A4 reduced the inhibitory effects of overexpression of miR-15a on cell proliferation. Therefore, circSLC26A4 may suppress the maturation of miR-15a in NSCLC to inhibit cancer cell proliferation.
\end{abstract}

\section{Introduction}

Non-small cell lung cancer (NSCLC) accounts for $>80 \%$ of lung cancer cases worldwide according to the statistics of global cancer in 2018 (1). Patients with NSCLC are usually treated with

Correspondence to: Dr Ji Liu, Department of Anesthesiology, Shanghai Pulmonary Hospital, 507 Zhengmin Road, Yangpu, Shanghai 200433, P.R. China

E-mail: jiliuanesthesiolog@163.com

Key words: non-small cell lung cancer, circular RNA solute carrier family 26 member 4, microRNA-15a, precursor, cell proliferation surgical resection, chemotherapy and radiotherapy, and surgical resection is only appropriate for patients diagnosed at early stages, and resistance usually develops during the long-term application of chemotherapy and radiotherapy $(2,3)$. With the emergence of targeted therapies, survival of certain NSCLC cases, especially patients diagnosed at advanced stages, has been markedly improved $(4,5)$. However, as the molecular mechanisms of NSCLC are unclear, there remains a lack of effective targets for targeted therapy $(6,7)$. Therefore, an improved understanding of the pathogenesis of NSCLC is required.

Most cases of NSCLC are associated with tobacco smoking (8), while $10 \%$ of NSCLC occurs in never-smokers (9), suggesting the involvement of other factors, such as molecular factors, in the pathogenesis of NSCLC.In effect, certain molecular signaling pathways, such as the TGF- $\beta$ and KRAS signaling pathways, have been demonstrated to be potential targets for targeted therapies of NSCLC, which can be performed to treat NSCLC by regulating the expression levels of NSCLC-related genes $(10,11)$. Circular RNAs (circRNAs/circs) are single strand RNA transcripts closed by covalent bonds (12). CircRNAs have limited protein-coding capacity; however, they are involved in cancer biology by regulating gene expression, suggesting that circRNAs are potential therapeutic targets for NSCLC (13). In a recent study, circRNA solute carrier family 26 member 4 (circSLC26A4) has been reported to be an oncogene in cervical cancer (14); however, to the best of our knowledge, its role in NSCLC is unknown. Our preliminary microarray analysis revealed altered expression levels of circSLC26A4 in NSCLC and its inverse correlation with mature microRNA (miRNA/miR)-15a, which is a cancer-related miRNA (15). The present study aimed to explore the potential interaction between circSLC26A4 and miR-15a in NSCLC.

\section{Materials and methods}

Tissue collection. NSCLC tissues and adjacent non-tumor tissues (within $5 \mathrm{~cm}$ around tumors) were collected from 64 patients with NSCLC who underwent surgical resection [40 male and 24 female patients; 28 cases of lung adenocarcinoma (LUAD) and 36 cases of lung squamous cell carcinoma (LUSC)] at Shanghai Pulmonary Hospital (Shanghai, China) between June 2016 and May 2019. The patients had an age range of 45-69 years, with a median age of 56 years. The present study was approved by the Ethics Committee of 
Shanghai Pulmonary Hospital, Tongji University (Shanghai, China). Patients who had severe clinical disorders or initiated therapies were excluded from the present study. All patients signed the written informed consent form. Detailed information of the patients is presented in Table I.

Therapy and follow-up. The 64 patients with NSCLC were classified into American Joint Committee on Cancer stage I or II $(n=26)$ and III or IV $(n=38)(16)$. The 64 patients were treated with surgical resection, radiotherapy, chemotherapy or immunotherapy according to their cancer stage and health conditions. The patients were followed up in a monthly manner until July 2020. Patient survival conditions were recorded and survival analysis was performed. All patients completed the follow-up or died of NSCLC during the follow-up.

NSCLC cells and transfection. The two human NSCLC cell lines, H1793 (LUAD) and DMS 79 (LUSC), obtained from American Type Culture Collection were used in the present study. Cells were cultured in a mixture composed of 90\% RPMI-1640 medium (HyClone; Cytiva) and 10\% FBS (Gibco; Thermo Fisher Scientific, Inc,) with $100 \mathrm{U} / \mathrm{ml}$ penicillin and $100 \mu \mathrm{g} / \mathrm{ml}$ streptomycin. The cell culture conditions were: $37^{\circ} \mathrm{C}, 5 \% \mathrm{CO}_{2}$ and $95 \%$ humidity. Cells were collected at a confluence rate of $\sim 85 \%$ for the subsequent experiments. Vector expressing circSLC26A4 was constructed with pcDNA3.1(+) CircRNA Mini Vector (Addgene, Inc.) as the backbone. To overexpress miR-15a, miR-15a mimic (5'-UAGCAGCACAUA AUGGUUUGUG-3') and non-specific miRNA [5'-UUCUCC GAACGUGUCACGUTT-3'; used as the negative control (NC)] were purchased from Sigma-Aldrich (Merck KGaA). H1793 and DMS 79 cells were counted and $5 \times 10^{7}$ cells were transfected with $45 \mathrm{nM}$ miRNA or $1 \mu \mathrm{g}$ expression vector using Lipofectamine $^{\circledR} 2000$ (Invitrogen; Thermo Fisher Scientific, Inc.) at $37^{\circ} \mathrm{C}$ for $24 \mathrm{~h}$. Empty vector- or miRNA NC-transfections were performed to serve as NC experiments. Untransfected cells were used as the control cells. Prior to the subsequent assays, cells were cultured in fresh medium for another $48 \mathrm{~h}$.

RNA preparation. Ribozol (Invitrogen; Thermo Fisher Scientific, Inc.) was used to extract total RNA from tissue samples and H1793 and DMS 79 cells. RNA samples were digested with DNase I at $37^{\circ} \mathrm{C}$ for $2 \mathrm{~h}$ to remove genomic DNAs. RNA integrity was checked using 6\% urea-PAGE gel electrophoresis. The optical density (OD)260/280 ratio of RNA sample was measured to reflect RNA integrity using NanoDrop 1000 instrument spectrophotometer (Thermo Fisher Scientific, Inc.).

Reverse transcription-quantitative PCR (RT-qPCR). Total RNA (500 ng) was reverse transcribed into cDNA using PrimeScript RT Master Mix (Takara Biotechnology Co., Ltd.) according to the manufacturer's protocol. SYBR Green Master Mix (Bio-Rad Laboratories, Inc.) was used to perform qPCR. The expression levels of circSLC26A4 were determined using GAPDH as the internal control. Expression levels of mature miR-15a and miR-15a precursor were analyzed using the All-in-One ${ }^{\mathrm{TM}}$ miRNA qRT-PCR Reagent kit (GeneCopoeia, Inc.) according to the manufacturer's protocol. Sequence-specific forward and reverse primers were used to perform RT and qPCR to determine the expression levels of miR-15a precursor.
Table I. Clinicopathological characteristics of patients with non-small cell lung cancer $(n=64)$.

\begin{tabular}{ll}
\hline Characteristics & No. \\
\hline Age, years & \\
$\leq 55$ & 34 \\
$>55$ & 30 \\
Sex & \\
Male & 40 \\
Female & 24 \\
Smoking history & \\
Smoker & 39 \\
Nonsmoker & 25 \\
Pathological pattern & \\
LUAD & 28 \\
LUSC & 36 \\
Lymphatic metastasis & \\
No & 24 \\
Yes & 40 \\
Stage & \\
I+II & 26 \\
III+IV & 38
\end{tabular}

LUAD, lung adenocarcinoma; LUSC, lung squamous cell carcinoma.

To measure the expression levels of mature miR-15a, poly (A) addition was first performed, followed by using poly (T) as the reverse primer to perform both RT and qPCR. The conditions of PCR reaction were initial denaturation at $95^{\circ} \mathrm{C}$ for $10 \mathrm{~min}$, followed by 40 cycles at $95^{\circ} \mathrm{C}$ for $15 \mathrm{sec}$ and $60^{\circ} \mathrm{C}$ for $30 \mathrm{sec}$. The expression levels of miRNAs were normalized to the internal reference U6. All experiments were performed in three technical replicates. The $2^{-\Delta \Delta C q}$ method was used to normalize $\mathrm{Ct}$ values of target genes to the corresponding internal control (17). The primers used in the present study were: CircSLC26A4 forward. 5'-TCCAAGTGCTGGTCTCACAG-3' and reverse, 5'-CCATAT CCGACAGGAACTGC-3'; miR-15a precursor forward, 5'-GCC GAGTAGCAGCACACATAA-3' and reverse, 5'-CAGTGCGTG TCGTGGAGT-3'; mature miR-15a forward, 5'-TAGCAGCAC ATAATGG-3' and reverse, 5'-GTGCAGGGTCCGAGGT-3'; U6 forward, 5'-CTCGCTTCGGCAGCACA-3' and reverse, 5'-AACGCTTCACGAATTTGCGT-3'; and GAPDH forward, 5'-ATCACTGCCACCCAGAAGAC-3' and reverse, 5'-TTTCTA GACGGCAGGTCAGG-3'.

Cell Counting Kit-8 (CCK-8) assay. Transfected H1793 and DMS 79 cells were subjected to cell proliferation analysis using a CCK-8 assay (Sigma-Aldrich; Merck KGaA). Briefly, $0.1 \mathrm{ml}$ medium containing 4,500 cells was added into each well of a 96-well plate. H1793 and DMS 79 cells were cultured at $37^{\circ} \mathrm{C}$ for $24,48,72$ and $96 \mathrm{~h}$, and then $10 \mu \mathrm{l} \mathrm{CCK}-8$ regent was added for $2 \mathrm{~h}$. The measurement of OD values was performed, and the absorbance was detected at $450 \mathrm{~nm}$.

Statistical analysis. SPSS 22.0 (IBM Corp.) was used to conduct the statistical analysis of data. The expression levels 
A

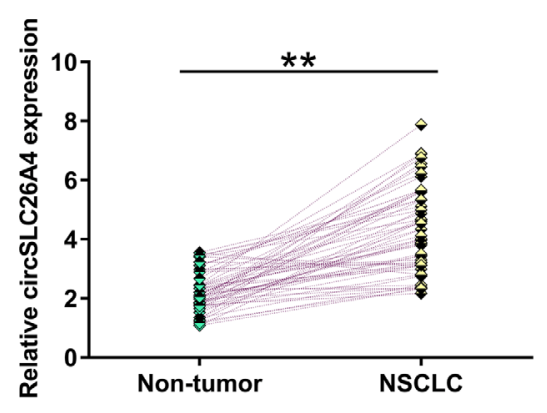

C

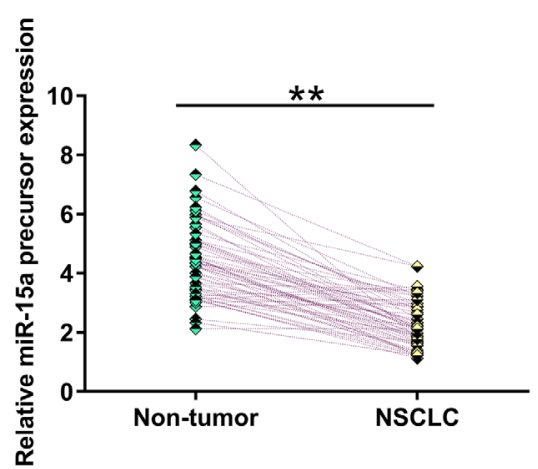

B

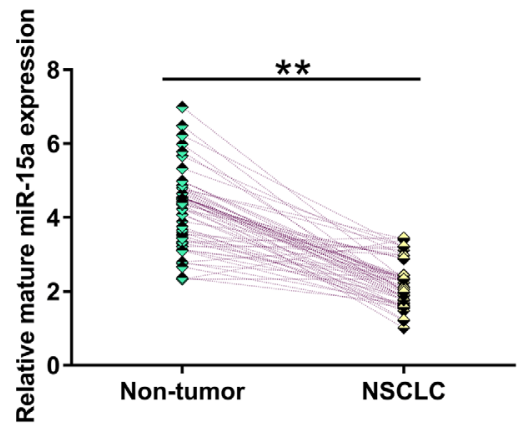

Figure 1. Altered expression levels of circSLC26A4, mature miR-15a and miR-15a precursor in NSCLC tissues. NSCLC tissues and adjacent non-tumor tissues were harvested from 64 patients with NSCLC, followed by the determination of the expression levels of (A) circSLC26A4, (B) mature miR-15a and (C) miR-15a precursor in these tissue samples by reverse transcription-quantitative PCR. The expression levels of circSLC26A4, mature miR-15a and miR-15a precursor in NSCLC and non-tumor tissues were presented as average values of three technical replicates and data comparisons were performed using a paired $t$-test. ${ }^{* *} \mathrm{P}<0.01$. circSLC26A4, circular RNA solute carrier family 26 member 4; miR-15a, microRNA-15a; NSCLC, non-small cell lung cancer.

of circSLC26A4, mature miR-15a and miR-15a precursor in NSCLC and non-tumor tissues were presented as average values of three technical replicates. Data comparisons were performed using a paired t-test. Other data on cell transfection, RT-qPCR and CCK-8 assay were presented as the mean \pm SD of three biological replicates. The comparisons of the study groups was conducted using one-way ANOVA followed by Tukey's post hoc test. Survival analysis was performed by dividing the 64 patients with NSCLC into high and low circSLC26A4 groups ( $n=32$; cutoff value, median expression level of circSLC26A4 in NSCLC tissues), followed by the plotting of Kaplan-Meier survival curves and the use of a log-rank test to compare survival curves. Pearson's correlation coefficient was used for correlation analysis. $\mathrm{P}<0.05$ was considered to indicate a statistically significant difference.

\section{Results}

Altered expression levels of circSLC26A4, mature miR-15a and miR-15a precursor in NSCLC tissues. The expression levels of circSLC26A4, mature miR-15a and miR-15a precursor in NSCLC tissues and adjacent non-tumor tissues were measured by RT-qPCR. The results revealed that, compared with that in non-tumor tissues, circSLC26A4 expression was significantly upregulated in NSCLC tissues (Fig. 1A; P<0.01). Furthermore, the expression levels of mature miR-15a (Fig. 1B) and miR-15a precursor (Fig. 1B) were significantly downregulated in NSCLC tissues compared with in non-tumor tissues $(\mathrm{P}<0.01)$. Therefore, circSLC26A4 and miR-15a may be involved in NSCLC.

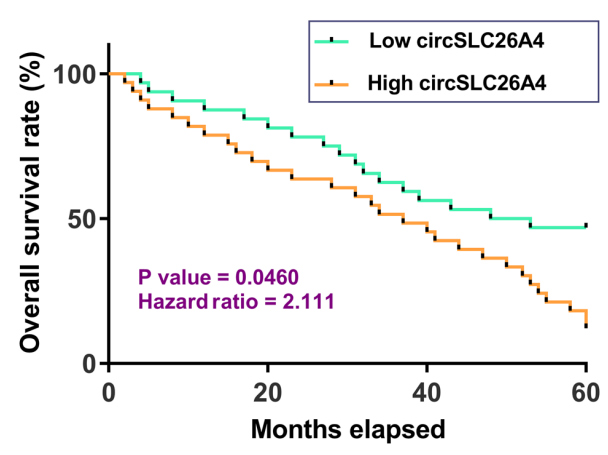

Figure 2. High expression levels of circSLC26A4 in NSCLC tissues are associated with poor survival of patients with NSCLC. Survival analysis was carried out by dividing the 64 patients with NSCLC into high and low circSLC26A4 groups ( $n=32$; cutoff value, median expression level of circSLC26A4 in NSCLC tissues), followed by the plotting of survival curves and the use of a log-rank test to compare the survival curves. circSLC26A4, circular RNA solute carrier family 26 member 4; NSCLC, non-small cell lung cancer.

High expression levels of circSLC26A4 in NSCLC tissues are associated with poor survival of patients with NSCLC. Survival curve analysis revealed that, compared with patients in the low circSLC26A4 expression group, patients in the high circSLC26A4 expression group had a significantly lower overall survival rate. Therefore, high expression levels of circSLC26A4 may predict poor survival of patients with NSCLC (Fig. 2).

circSLC26A4 suppresses the maturation of miR-15a in NSCLC. Pearson's correlation coefficient analysis was used to determine 


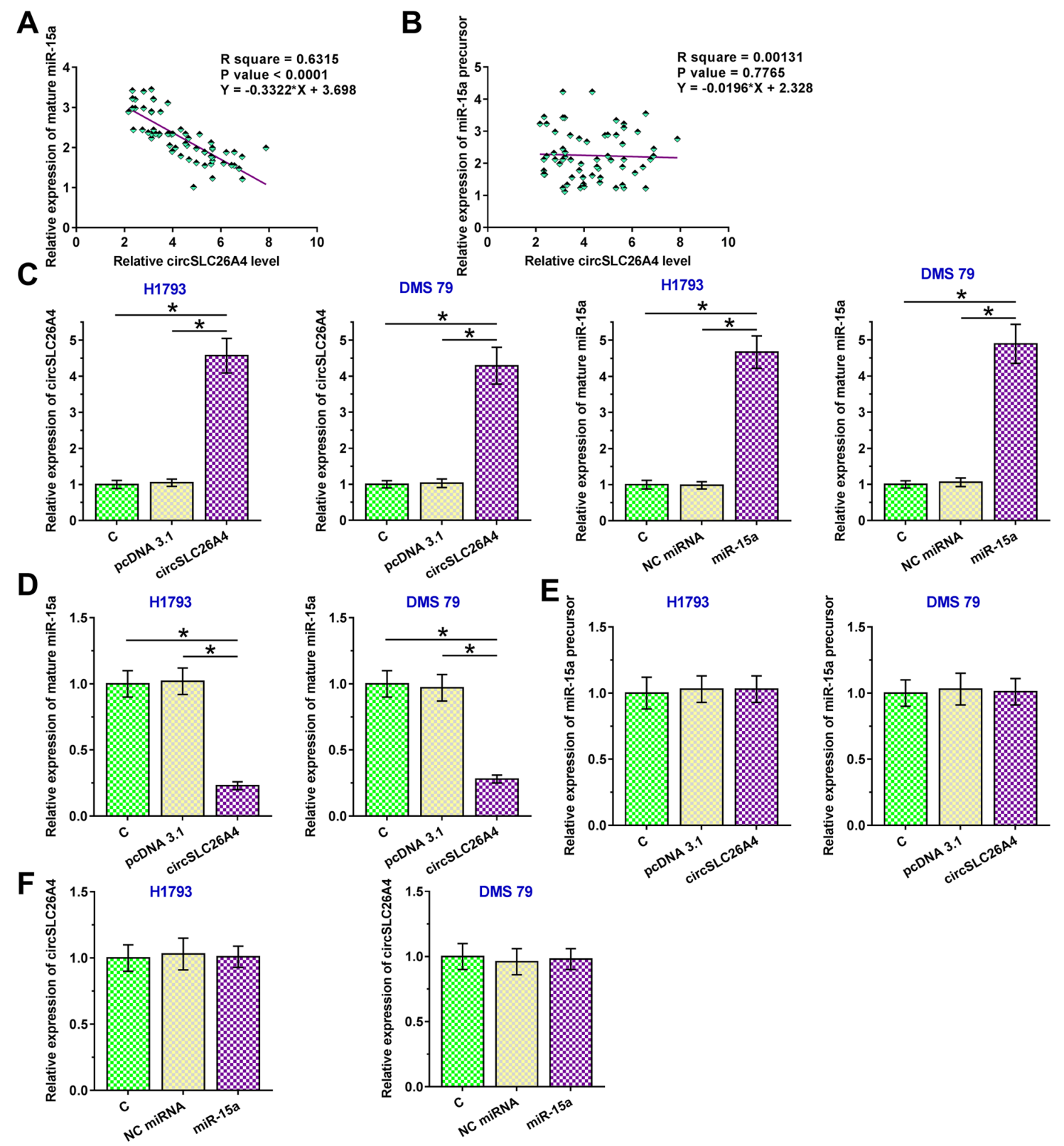

Figure 3. circSLC26A4 suppresses the maturation of miR-15a in NSCLC. Pearson's correlation coefficient was used to analyze the correlations between the expression levels of circSLC26A4 and (A) mature miR-15a or (B) miR-15a precursor in NSCLC tissues. (C) To further explore the interaction between circSLC26A4 and miR-15a, circSLC26A4 expression vector or miR-15a mimic was transfected into H1793 and DMS 79 cells, and transfection efficiency was confirmed at $48 \mathrm{~h}$ after transfection. The effects of overexpression of circSLC26A4 on (D) mature miR-15a and (E) miR-15a precursor expression, and (F) the effects of overexpression of miR-15a on the expression levels of circSLC26A4 were also analyzed by reverse transcription-quantitative PCR. Data of three biological replicates of cell transfection experiments are presented as the mean $\pm \mathrm{SD}$, and one-way ANOVA followed by Tukey's post-hoc test was used for data comparisons of study groups. "P<0.05. C, control (untransfected cells); circSLC26A4, circular RNA solute carrier family 26 member 4; miR-15a, microRNA-15a; miRNA, microRNA; NC, negative control; NSCLC, non-small cell lung cancer.

the correlation between circSLC26A4 expression and mature miR-15a or miR-15a precursor expression in NSCLC tissues. It was observed that circSLC26A4 expression was inversely and significantly correlated with the expression levels of mature miR-15a in NSCLC tissues (Fig. 3A). However, there was no significant correlation between the expression levels of circSLC26A4 and miR-15a precursor (Fig. 3B). To further explore the interaction between them, circSLC26A4 and miR-15a were overexpressed in both $\mathrm{H} 1793$ and DMS 79 cells $(\mathrm{P}<0.05$; Fig. 3C). Overexpression of circSLC26A4 resulted in downregulation of mature miR-15a expression $(\mathrm{P}<0.05$; Fig. 3D), but not in downregulation of miR-15a precursor expression (Fig. 3E).
These findings suggested that the maturation of miR-15a in NSCLC cells could be suppressed by circSLC26A4. By contrast, overexpression of miR-15a exhibited no significant effect on the expression levels of circSLC26A4 (Fig. 3F).

Overexpression of circSLC26A4 promotes NSCLC cell proliferation via miR-15a. A CCK-8 assay was performed to explore the role of circSLC26A4 and miR-15a in regulating the proliferation of H1793 and DMS 79 cells. Overexpression of circSLC26A4 significantly increased NSCLC cell proliferation, while overexpression of miR-15a decreased cell proliferation compared with their respective NCs. In addition, overexpression 

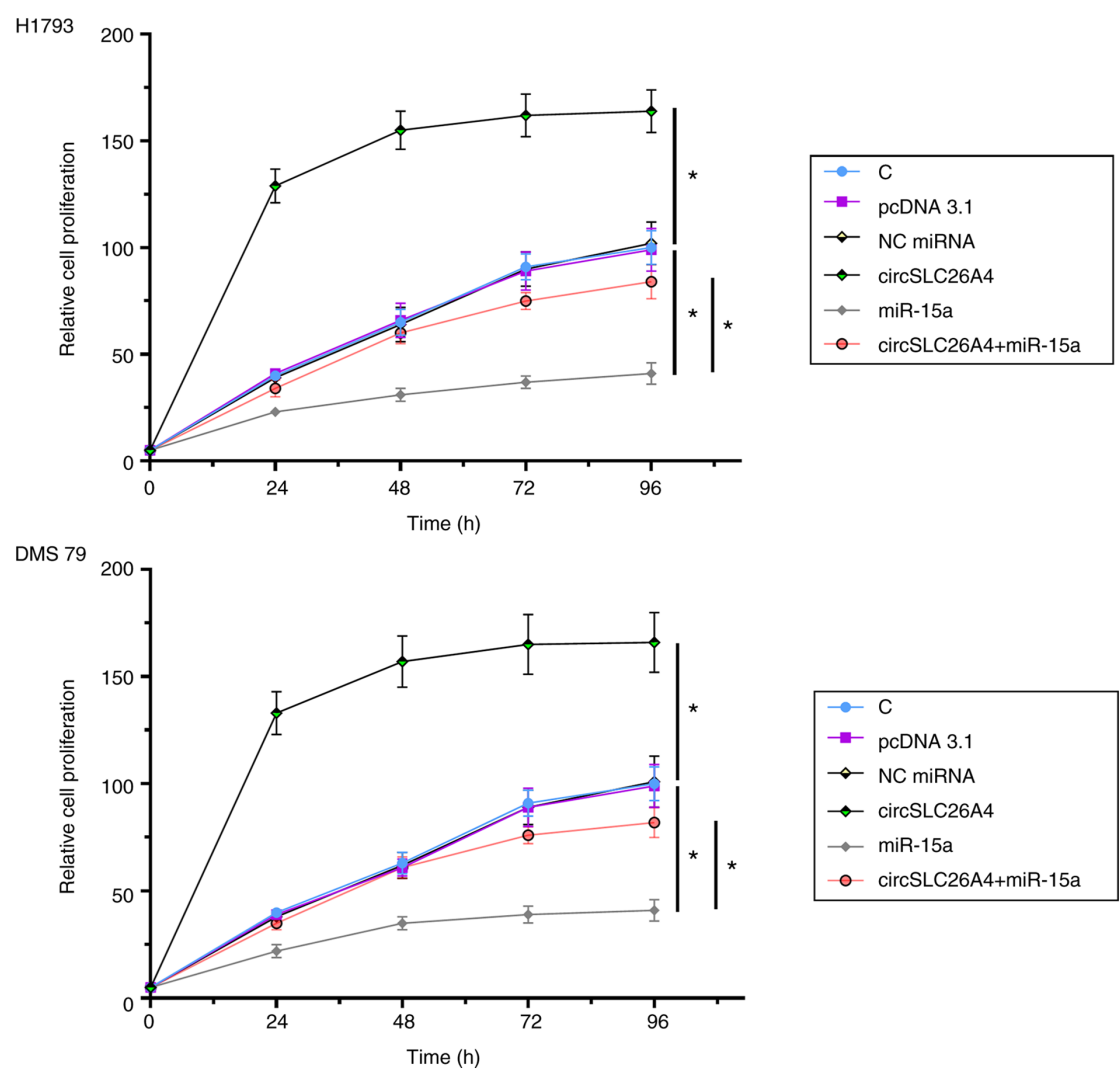

Figure 4. Overexpression of circSLC26A4 promotes non-small cell lung cancer cell proliferation via miR-15a. A Cell Counting Kit-8 assay was performed to analyze the roles of circSLC26A4 and miR-15a in regulating the proliferation of H1793 and DMS 79 cells. Data of three biological replicates of cell transfection experiments are presented as the mean $\pm \mathrm{SD}$, and one-way ANOVA followed by Tukey's post-hoc test was used for data comparisons of study groups. ${ }^{*}<0.05$. C, control (untransfected cells); circSLC26A4, circular RNA solute carrier family 26 member 4; miR-15a, microRNA

of circSLC26A4 reduced the inhibitory effects of overexpression of miR-15a on cell proliferation $(\mathrm{P}<0.05$; Fig. 4).

\section{Discussion}

The differential expression of circSLC26A4 in NSCLC and its interaction with miR-15a were investigated in the present study. The present data demonstrated that circSLC26A4 expression was significantly upregulated in NSCLC tissues and it could promote the proliferation of NSCLC cells by suppressing the maturation of miR-15a.

In a recent study, circSLC26A4 expression was reported to be upregulated in cervical cancer, and its high expression was significantly associated with poor survival of patients with cervical cancer (14). In addition, circSLC26A4 promotes the migration and proliferation of cervical cancer cells by sponging miR-1287-5p to upregulate HOXA7 (14). However, the regulatory effects of circSLC26A4 in NSCLC is still unclear. In the present study, to investigate the role of circSLC26A4 in the development of NSCLC, the expression levels of circSLC26A4 in NSCLC and adjacent non-tumor tissues were measured. circSLC26A4 expression was upregulated in NSCLC tissues. In addition, overexpression of circSLC26A4 increased the proliferation of both LUAD and LUSC cells, which are the two major subgroups of NSCLC. Therefore, circSLC26A4 may promote the proliferation of cancer cells, resulting in the development and progression of NSCLC.
Even with advances in the diagnosis and treatment of NSCLC, the prognosis of NSCLC is still poor $(18,19)$. Goyal et al (19) conducted a retrospective study of patients with NSCLC diagnosed between 2004 and 2014 using the National Cancer Database, revealing that the overall 5-year survival rate of patients with stage IV NSCLC is only $24 \%$. This is mainly due to the low early diagnostic rate (19). Furthermore, due to the lack of sensitive markers, the early diagnosis of NSCLC is unlikely to be markedly improved in the near future (20). The present study demonstrated that high expression levels of circSLC26A4 were closely associated with poor survival of patients, suggesting a potential role of circSLC26A4 as a prognostic biomarker for NSCLC. Therefore, evaluation of the expression levels of circSLC26A4 prior to therapy may assist the determination of treatment approaches, which would in turn improve patient survival. miR-15a has been reported to be a tumor suppressor in different types of cancer, including NSCLC $(21,22)$. One study reported that miR-15a expression was downregulated in NSCLC tissues and cells, and overexpression of miR-15a inhibited NSCLC cell proliferation, migration and invasion (23). Another study revealed that miR-15a expression is markedly downregulated in NSCLC tissues, and overexpression of miR-15a markedly suppresses cell viability, invasion and migration, and accelerates the apoptosis of NSCLC cells (24). miR-15a not only suppresses tumor metastasis, but also increases the sensitivity of cancer cells to chemotherapy $(19,20)$. Consistently, the present study revealed the downregulation of miR-15a expression in NSCLC and its 
inhibitory effects on cell proliferation. Notably, the present study revealed that overexpression of circSLC26A4 suppressed the maturation of miR-15a in NSCLC cells. However, to the best of our knowledge, the underlying mechanism is unknown. To be cleaved into mature miR-15a, miR-15a precursor should be transported out of nucleus to enter the cytoplasm (25). The involvement of circSLC26A4 in the transportation of miR-15a will be explored in our future studies.

In conclusion, circSLC26A4 expression is upregulated in NSCLC and may promote cancer cell proliferation by suppressing the maturation of miR-15a. The current findings may help to increase the understanding of NSCLC pathogenesis and to identify potential targets for the treatment of patients with NSCLC.

\section{Acknowledgements}

Not applicable.

\section{Funding}

The present study was supported by Shanghai Science and Technology Commission Animal Experiment Model Project (grant no. 19140904102).

\section{Availability of data and materials}

The datasets used and/or analyzed during the current study are available from the corresponding author on reasonable request.

\section{Authors' contributions}

QC conducted the experiments and contributed to data analysis and manuscript writing. JL provided critical guidance for this manuscript, designed this study, interpreted data, revised this manuscript, and gave final approval of the version to be published. HL contributed to data analysis and interpretation. All authors confirmed the authenticity of the data and read and approved the final manuscript.

\section{Ethics approval and consent to participate}

This study was approved by the Ethics Committee of Shanghai Pulmonary Hospital, Tongji University (Shanghai, China), and all patients provided written informed consent.

\section{Patient consent for publication}

Not applicable.

\section{Competing interests}

The authors declare that they have no competing interests.

\section{References}

1. Adjei AA: Lung cancer worldwide. J Thorac Oncol 14: 956, 2019.

2. Goldstraw P, Ball D, Jett JR, Le Chevalier T, Lim E, Nicholson AG and Shepherd FA: Non-small-cell lung cancer. Lance 378: 1727-1740, 2011.

3. Jamal-Hanjani M, Wilson GA, McGranahan N, Brikbak N, Watkins TBK, Veeriah S, Shafi S, Johnson DH, Mitter R, Rosenthal R, et al: Tracking the evolution of non-small-cell lung cancer. N Engl J Med 376: 2109-2121, 2017.
4. Chan BA and Hughes BG: Targeted therapy for non-small cell lung cancer: Current standards and the promise of the future. Transl Lung Cancer Res 4: 36-54, 2015.

5. Janku F, Stewart DJ and Kurzrock R: Targeted therapy in non-small-cell lung cancer-is it becoming a reality? Nat Rev Clin Oncol 7: 401-414, 2010.

6. Herbst RS, Morgensztern D and Boshoff C: The biology and management of non-small cell lung cancer. Nature 553: 446-454, 2018

7. Hirsch FR, Suda K, Wiens J and Bunn PA Jr: New and emerging targeted treatments in advanced non-small-cell lung cancer. Lancet 388: 1012-1024, 2016.

8. Joseph AM, Rothman AJ, Almirall D, Begnaud A, Chiles C, Cinciripini PM, Fu SS, Graham AL, Lindgren BR, Melzer AC, et al: Lung cancer screening and smoking cessation clinical trials. SCALE (smoking cessation within the context of lung cancer screening) collaboration. Am J Respir Crit Care Med 197: 172-182, 2018.

9. Saito S, Espinoza-Mercado F, Liu H, Sata N, Cui X and Soukiasian HJ: Current status of research and treatment for non-small cell lung cancer in never-smoking females. Cancer Biol Ther 18: 359-368, 2017.

10. Eser PÖ and Jänne PA: TGF $\beta$ pathway inhibition in the treatment of non-small cell lung cancer. Pharmacol Ther 184: 112-130, 2018.

11. Tomasini P, Walia P, Labbe C, Jao K and Leighl NB: Targeting the KRAS pathway in non-small cell lung cancer. Oncologist 21: 1450-1460, 2016.

12. Zhou R, Wu Y, Wang W, Su W, Liu Y, Wang Y, Fan C, Li X, Li G, Li Y, et al: Circular RNAs (circRNAs) in cancer. Cancer Lett 425: 134-142, 2018

13. Meng S, Zhou H, Feng Z, Xu Z, Tang Y,Li P and Wu M: CircRNA: Functions and properties of a novel potential biomarker for cancer. Mol Cancer 16: 94, 2017.

14. Ji F, Du R, Chen T, Zhang M, Zhu Y, Luo X and Ding Y: Circular RNA circSLC26A4 accelerates cervical cancer progression via miR-1287-5p/HOXA7 axis. Mol Ther Nucleic Acids 19: 413-420, 2020.

15. He J: Knocking down miR-15a expression promotes the occurrence and development and induces the EMT of NSCLC cells in vitro. Saudi J Biol Sci 24: 1859-1865, 2017.

16. Detterbeck FC, Boffa DJ, Kim AW and Tanoue LT: The eighth edition lung cancer stage classification. Chest 151: 193-203, 2017.

17. Livak KJ and Schmittgen TD: Analysis of relative gene expression data using real-timequantitative PCR and the 2(-Delta Delta C(T)) method. Methods 25: 402-408, 2001.

18. Stokes WA, Bronsert MR, Meguid RA, Blum MG, Jones BL, Koshy M, Sher DJ, Louie AV, Palma DA, Senan S, et al: Post-treatment mortality after surgery and stereotactic body radiotherapy for early-stage non-small-cell lung cancer. J Clin Oncol 36: 642-651, 2018.

19. Goyal G, Kommalapati A, Bartley AC, Gunderson TM, Adjei AA and Go RS: Association between hospital volume and mortality of patients with metastatic non-small cell lung cancer. Lung Cancer 122: 214-219, 2018.

20. Postmus PE, Kerr KM, Oudkerk M, Senan S, Waller DA, Vansteenkiste J, Escriu C and Peters S; ESMO Guidelines Committee: Early and locally advanced non-small-cell lung cancer (NSCLC): ESMO Clinical Practice Guidelines for diagnosis, treatment and follow-up. Ann Oncol 28 (Suppl-4): iv1-iv21, 2017.

21. Lan F, Yue X, Ren G, Li H, Ping L, Wang Y and Xia T: miR-15a/16 enhances radiation sensitivity of non-small cell lung cancer cells by targeting the TLR1/NF- $\mathrm{KB}$ signaling pathway. Int J Radiat Oncol Biol Phys 91: 73-81, 2015.

22. Bozok Çetintaş V, Tetik Vardarlı A, DüzgünZ, Tezcanlı Kaymaz B, Açıkgöz E, Aktuğ H, Kosova Can B, Gündüz C and Eroğlu Z: miR-15a enhances the anticancer effects of cisplatin in the resistant non-small cell lung cancer cells. Tumour Biol 37: 1739-1751, 2016.

23. Guo S, Li M, Li J and Lv Y: Inhibition mechanism of lung cancer cell metastasis through targeted regulation of Smad3 by miR-15a. Oncol Lett 19: 1516-1522, 2020.

24. Yang T, Thakur A, Chen T, Chen T, Yang L, Lei G, Liang Y, Zhang S, Ren H and Chen M: MicroRNA-15a induces cell apoptosis and inhibits metastasis by targeting BCL2L2 in non-small cell lung cancer. Tumour Biol 36: 4357-4365, 2015.

25. Pickering BF, Yu D and Van Dyke MW: Nucleolin protein interacts with microprocessor complex to affect biogenesis of microRNAs 15a and 16. J Biol Chem 286: 44095-44103, 2011.

This work is licensed under a Creative Commons Attribution-NonCommercial-NoDerivatives 4.0 International (CC BY-NC-ND 4.0) License. 